\title{
Metodología de análisis de la condición del pavimento a partir del cuenco de deflexión
}

\section{Methodology of analysis of the condition of the pavement starting from the deflection bowl}

Ing. Carlos Andrade Neto

Profesor de la Facultad de Ciencias de la Universidad Agostino Neto, Angola.

carlosandneto@hotmail.com

\section{Dr. Ing. Iván Machado López}

Facultad de Construcciones de la Universidad Central Marta Abreu de las Villas, Cuba.

ivanm@uclv.edu.cu

\section{Dr. Ing. Jorge Rufino}

Profesor de la Facultad de Ciencias de la Universidad Agostino Neto, Angola.

jorgerufino2@gmail.com

Fecha de recepción: 14 de julio de 2014 / Fecha de aprobación: 24 de setiembre de 2014

\section{RESUMEN}

En el presente trabajo se expone una metodología que permite realizar una propuesta de intervención con respecto a la rehabilitación del pavimento, así como la determinación de los espesores de refuerzo, a partir de los parámetros del cuenco de deflexión.

Se obtienen, sobre la base de las estructuras reglamentadas en la norma SATCC, 2001 (Southern África Transport and Communications Commission), y el uso del programa de computación JULEA las correspondientes superficies de respuesta a partir de los índices del cuenco de deflexión, lo que permite formular las expresiones matemáticas para estimar el módulo de la subrasante, la condición de cada una de las capas de la estructura del pavimento y la ecuación para el cálculo del espesor de refuerzo.

Se muestran los resultados de la metodología aplicada en un caso de estudio, donde se realizan mediciones del cuenco de deflexión con la viga Benkelman y el uso del Deflectómetro de Impacto (FWD por sus siglas en inglés) verificándose la validez del procedimiento propuesto el cual incrementa la rapidez en la obtención de los resultados del diagnóstico y soluciones de rehabilitación.

PALABRAS ClAVE: Pavimentos flexibles, cuenco de deflexión, rehabilitación de carreteras.

\section{ABSTRACT}

Presently work is exposed a methodology that allows to carry out an intervention proposal with regard to the rehabilitation of the pavement, as well as the determination of the reinforcement thickness, starting from the parameters of the deflection bowl.

They are obtained, on the base of the structures regulated in the norm of SATCC, 2001 (Southern Africa Transport and Communications Commission), and the use of the computation program JULEA, the corresponding answer surfaces starting from the indexes of the deflection, what allows to formulate the mathematical expressions to estimate the module and the condition of each one of the layers of the structure of the pavement and the equation for the calculation of the thickness reinforcement.

The results of the methodology are shown applied in a study case, where they are carried out confirmations of the deflection with the Benkelman beam and the use of the Falling Weight Deflectometer (FWD), being verified the validity of the proposed procedure that increase the speed in the obtaining of the results of the diagnosis and rehabilitation solutions

KEY WORDS: Flexible pavements, deflection bowl, rehabilitation of roads. 


\section{INTRODUCCIÓN}

La generación de metodologías de proyectos de rehabilitación de pavimentos es una necesidad que se impone debido a la importancia de la recuperación y mantenimiento del patrimonio vial construido, dado que gran parte de las carteras construidas en Angola han sido construidas sobre la base de métodos empíricos, es necesario en estos momentos realizar evaluaciones de estas estructuras usando procedimientos racionales disponibles en programas computacionales con capacidad de simular las respuestas de los pavimentos a las cargas aplicadas, lo que permite considerando las condiciones locales establecer ecuaciones para la evaluación de la condición del pavimento.

En este sentido existe una gran variedad de métodos de rehabilitación de pavimentos, por lo tanto es necesario proponer una metodología para la adecuada toma de decisiones en cuanto a la rehabilitación de los pavimentos considerando los recursos materiales, ambientes y capacidades disponibles en el país; la norma SATCC (2001) resulta de preponderante utilización en la región, sin embargo la utilización de un método que pueda ser acompañado, verificado y sistemáticamente corregido es siempre mejor que adaptar de forma ciega cualquier normativa, por lo tanto sobre esta base es posible la obtención de una metodología para la rehabilitación de los pavimentos locales.

La metodología de retrocálculo (también denominada retroanálisis), es una herramienta que actualmente se considera la más adecuada para la evaluación estructural de los pavimentos, lo que permite la determinación de los módulos de las capas que la componen y de la subrasante con base en el conocimiento de los espesores y de los módulos iníciales.

Los métodos de retrocálculo pueden ser iterativos cuando realizan comparaciones sucesivas entre el cuenco de deflexión medido y el teórico, este último puede ser determinado por programas de computación, por el conjunto de estructuras evaluadas y almacenadas en un banco de datos o por regresión estadística, mientras los métodos simplificados son aquellos que se realizan por medio de la utilización de ecuaciones, tablas y gráficos basados en la teoría de elasticidad, este procedimiento es más rápido que los iterativos, pero con mucha menor precisión [1].

Con el retroanálisis es posible la introducción de ajustes para mejorar la confiabilidad de los métodos de diseño, esta herramienta se caracteriza por la determinación analítica de los módulos elásticos (de rigidez/resiliencia) de todas las capas del pavimento y de la subrasante a partir de la medición de los cuencos de deformación con el uso de instrumentos como la viga Benkelman, o con equipamientos tipo Deflectómetro de Impacto (FWD; Falling Weight Deflectometer por su denominación en inglés) [2].
En trabajos realizados para correlacionar los resultados que se obtienen con diferentes equipos de ensayos no destructivos, particularmente entre la viga Benkelman y el Deflectómetro de Impacto, puede encontrarse dispersión y no unicidad en las correlaciones de los resultados que se comparan [3], esto puede estar relacionado con diversos factores entre los cuales se destacan el conjunto de la propia estructura, las condiciones climáticas, el modo de aplicación de la carga, la metodología aplicada, la precisión intrínseca del equipo utilizado y la comprensión de la influencia de los errores de todo tipo involucrados.

Conociendo el espesor y los módulos de las capas del pavimento así como las cargas aplicadas, es posible por medio de la mecánica de los pavimentos determinar las tensiones, las deformaciones y los desplazamientos representados por la deflexión, con lo cual es posible realizar la evaluación y el diseño de las estructuras con vistas a su rehabilitación, en este sentido los métodos de retroanálisis permiten obtener los módulos en las condiciones de campo, con lo que se obtiene rapidez en la caracterización de los materiales minimizando en muchos casos la necesidad de toma de testigos o muestras, lo que puede redundar en economías para todo el proyecto.

Actualmente es posible establecer la condición estructural del pavimento a través de la medición del cuenco de deflexión, la medición de deflexiones brinda una forma rápida, eficiente, confiable y no destructiva de evaluar la condición estructural de un pavimento y la subrasante [4], de este modo los datos obtenidos pueden ser especialmente utilizados para el retrocálculo en el análisis de las propiedades de las capas en la evaluación de los pavimentos en servicio [5] [6].

Sin embargo a criterio del autor al aplicar los métodos de retrocálculo basados en fórmulas estadísticas predictivas obtenidas por regresiones, puede ser necesario precisar las condiciones del pavimento para el cual son válidas las expresiones formuladas [7].

Se debe considerar que las anteriores aplicaciones son posibles a partir de la deducción de algunos parámetros asociados a la medición de las deflexiones, los cuales indican el estado de la estructura del pavimento, estos parámetros se basan principalmente en la forma del cuenco de deflexiones, la deflexión máxima, el área del cuenco y el índice de curvatura superficial [4].

Diversos autores [8-11] presentan diferentes ecuaciones desarrolladas en que se utilizan parámetros tales como el Índice Base de Curvatura (ICB), Índice de Curvatura Superficial (ISC), Índice de la Capa Intermedia (ICI), considerando en algunos 
casos que estos índices pueden sustituir el retrocálculo. La norma aplicada en muchos casos en la región de estudio [12] presenta once parámetros del cuenco de deflexión incluyendo los anteriormente citados.

Las estructuras de la norma SATCC (2001) utilizadas son las referentes a las estructuras en bases granulares, de la evaluación realizada se verifica que los valores límites de los parámetros anteriormente indicados dependen del tráfico y del módulo de la subrasante, siendo estos dos parámetros utilizados para la propuesta de ecuaciones para el cálculo de valores limites que definen para la región angolana la condición del pavimento.

En este sentido para la obtención de los cuencos de deflexión se utiliza el programa de código abierto denominado JULEA (Jacob Uzan Layered Elastic Analysis), desarrollado por UZAN (1978) que utiliza el cálculo analítico con base en la teoría elástica lineal utilizando la solución de Burmister según propone [13].

De igual modo en el presente trabajo se utiliza también el programa computacional Alize desarrollado por el laboratorio central de puentes y carreteras de Francia, este es un método racional reglamentario de diseño de pavimentos de carreteras en el país europeo, el mismo utiliza el modelo matemático elástico lineal para obtener la respuesta en tensiones, deformaciones y deflexiones en las capas del pavimento y en la subrasante.

En las evaluaciones realizadas sobre la base de las técnicas de cómputo, las capas son consideradas horizontales, paralelas entre sí, de espesor constante, continuamente apoyadas entre sí con adherencia total, siendo el conjunto un medio elástico lineal, homogéneo, isótropo y continuo. Para definir la relación tensión deformación son necesarios solamente el módulo de Young (E), y el coeficiente de poisson $(\mathrm{U}<)$.

En general los catálogos para el diseño de pavimentos consisten en procedimientos basados en las condiciones locales y su aplicación fuera de la zona en que fueron elaborados necesariamente implica la realización de estudios de adaptación.

De ahí que el objetivo del presente trabajo es la adecuación de la norma SATCC (2001) a las condiciones de Angola a partir del empleo del método del retroanálisis teniendo en cuenta la toma de datos no destructivos que permitan sobre la base de la metodología que se propone obtener las exigencias de la estructura del pavimento para una adecuada rehabilitación.

\section{METODOLOGÍA}

Para el análisis de la condición del pavimento a partir de las deflexiones es necesario verificar si los resultados que se obtienen al considerar las estructuras propuestas en el catálogo de la norma SATCC (2001) son semejantes con los valores que se obtienen al aplicar la siguiente ecuación del módulo equivalente [14].

$$
E e q=\frac{\sum_{i=1}^{n} E i^{*} h i}{\sum_{i=1}^{n} h l}
$$

Siendo:

Eeq: módulo del pavimento equivalente;

Ei- módulo de la capa i;

$\sum_{i=1}^{n} h i$ espesor del pavimento equivalente;

hi: espesor de la capa;

i: numero de capas de la estructura del pavimento.

En primer lugar se introducen los valores de las estructuras del catálogo en el programa computacional Alize, se adopta para el módulo de la capa asfáltica el valor de $1000 \mathrm{MPa}$ correspondiente al valor más crítico calculado en función de la más alta temperatura considerada para las condiciones de Angola [7], de este modo se realiza la determinación de la deflexión máxima y el radio de curvatura correspondiente a la estructura completa presentada en la norma para la región húmeda, atendiendo al clima tropical húmedo preponderante en el país (denominado en la norma W-región húmeda).

Las estructuras equivalentes calculadas según la fórmula propuesta por Vila (1985) se introducen en el programa computacional Alize obteniendo de igual modo para este caso la deflexión máxima y el radio de curvatura.

De este modo se propone obtener una ecuación de la estructura equivalente en función del tráfico y la subrasante para esto se utiliza el programa Excel en el cual se introducen el espesor y el módulo equivalente. Como resultado se obtiene una familia de curvas que definen una superficie de respuesta dadas por una función linear por ejemplo del tipo siguiente en que $\mathrm{X}$ representa el tráfico y Y el valor que se pretende determinar.

$$
Y=a i * Z^{b i}
$$

Siendo: Y el término dependiente cuyo valor se pretende determinar, aii y bi son los coeficientes cuyas ecuaciones se han de obtener en función del parámetro concreto de entrada que para este caso particular representa el módulo de la subrasante.

La determinación de los coeficientes ai y bi en cada caso se realiza a partir de las ecuaciones lineares como por ejemplo del tipo siguiente: 


$$
a i=c^{*} X 1^{d} \quad \text { (3) } \quad \mathrm{bi}=\mathrm{e}^{*} \mathrm{X} 1+\mathrm{f}
$$

Remplazando estos coeficientes en la ecuación genérica del tipo $Y=a i * X 2^{b i}$ anteriormente descrita, resulta en la ecuación general necesaria para el cálculo del parámetro pretendido del tipo siguiente:

$$
\mathrm{Y}=\left(\mathrm{c} * \mathrm{X} 1^{\mathrm{d}}\right) * \mathrm{X}^{\left(\mathrm{e}^{*} \mathrm{X} 1+\mathrm{f}\right)}
$$

En la figura 1 se muestra la obtención de la ecuación $Y=h=a i * X^{b i}$ a partir de la superficie de respuesta en función de los parámetros de entrada $\mathrm{X}=\mathrm{N}$ y $\mathrm{X} 1=\mathrm{Es}$.

Utilizando el procedimiento descrito se determinan los parámetros necesarios para el cálculo de la estructura del pavimento en términos del espesor equivalente y el módulo equivalente. Se ha establecido que el procedimiento anteriormente descrito igualmente puede ser utilizado para la obtención de ecuaciones que permiten realizar la evaluación de la condición del pavimento en función de la deflexión máxima y el radio de curvatura, con lo cual se puede estimar el módulo de la subrasante, el espesor y el módulo equivalente. En este caso las deflexiones máximas y radios de curvaturas utilizados para la obtención de la ecuación son las obtenidas de las estructuras de la norma utilizando el programa Alize.

Sin embargo, para la determinación del cuenco de deflexión no resulta adecuado el programa Alize, de este modo los valores de las estructuras del catálogo, según se encuentran agrupadas, se introducen en el programa JULEA determinando los respectivos cuencos de deflexión, con lo cual se establecen las ecuaciones para el cálculo de los valores límites de la deflexión máxima y de los índices Índice de la Capa Superficial (ICS), Índice de la Capa Baja (ICB) y el Índice de la Capa Intermedia (ICI) con base en el tráfico previsto y el módulo de la subrasante, este último es estimado a partir de la ecuación obtenida de la correlación entre los valores de la norma y las deflexiones D120, o sea las deflexiones obtenidas en el punto a $120 \mathrm{~cm}$ del centro de aplicación de la carga presentada en los cuencos obtenidos con el programa JULEA.

En cada caso la ecuación para el cálculo concreto de cada parámetro adopta la forma correspondiente a la función de correlación que más se ajusta en dependencia de los datos de entrada, los resultados obtenidos con cada ecuación concreta se comparan con los datos iniciales determinando el valor del error y consecuentemente se determina la validez de la ecuación.

La condición estructural de la carretera puede estar en función de los parámetros deflectométricos [11], en ese sentido en este trabajo se propone realizar la evaluación de la estructura existente y la

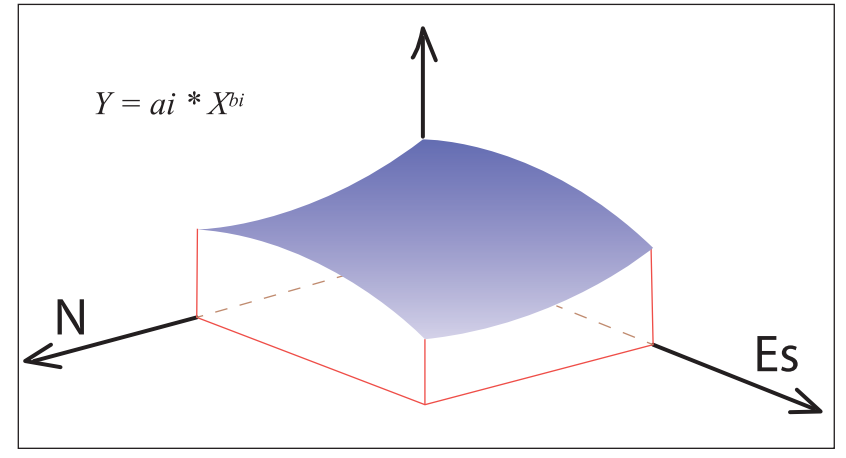

Figura 1. Superficie de respuesta para la obtención de las correspondientes ecuaciones

correspondiente recomendación en cuanto a su rehabilitación, para lo cual se compara la estructura existente con la esperada resultante de la determinación del espesor total del pavimento equivalente (h) y del módulo del pavimento equivalente (E), en función del tráfico esperado y la deflexión característica para cada tramo homogéneo, con la ecuación del pavimento equivalente se determina el espesor de refuerzo resultante de la diferencia entre la estructura esperada y la estructura existente.

Resulta conveniente establecer que la aplicación de las ecuaciones encontradas según la presente metodología posee dos salidas: la primera para el diagnóstico basada en la evaluación visual y los datos de campo de la deflexión que se obtienen con la Viga Benkelman y otra salida para realizar el diagnostico basado en los datos de campo que se obtienen sobre la base de la evaluación con el Deflectómetro de Impacto (FWD por sus siglas en ingles), este procedimiento es consistente con la teoría y con la bibliografía consultada [15].

\section{RESULTADOS}

\section{Validez de la utilización de la ecuación del pavimento equivalente}

Para probar la validez de la utilización de la ecuación del pavimento equivalente se correlacionan los valores de la deflexión máxima de la estructura calculados mediante el programa Alize, se tienen en cuenta los valores de la deflexión procedentes de las estructuras de la norma y la deflexión procedente de las estructuras calculadas a partir de la ecuación de la estructura equivalente.

En la figura 2 se muestra que existe una fuerte correlación entre estas variables, dado por $\mathrm{R}^{2}$, por lo que se reitera la correcta decisión del empleo de la ecuación propuesta por Vila (2005) para el análisis y comparación de las estructuras de la norma SATCC. 


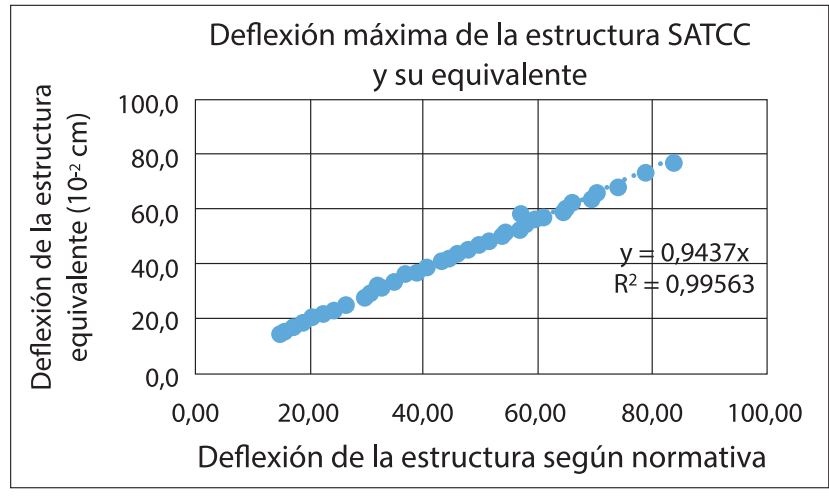

Figura 2. Correlación entre la deflexión del pavimento equivalente según la ecuación de Vila (2005) y la deflexión de la estructura del pavimento según la norma (SATCC)

\section{Expresiones matemáticas para ser usadas a partir de datos de campo obtenidos con la viga Benkelman}

Según la metodología antes descrita se obtienen las ecuaciones que permiten determinar el espesor total del pavimento equivalente de la estructura, al introducir el tráfico y el módulo de la subrasante, estos parámetros permiten realizar el cálculo de la estructura del pavimento.

$$
\begin{gathered}
h=(65.115 * \operatorname{Ln}(N)+1027.098) * E s^{(0.04 * \operatorname{Ln}(N)-0.476} \\
E=380.4 * N^{(-0.06) * E S}{ }^{0.236 * N^{0.07}}
\end{gathered}
$$

Siendo:

h: Espesor total del pavimento equivalente;

E: Módulo del pavimento equivalente;

Es: Módulo de la subrasante obtenido de la evaluación del suelo existente;

N: Tráfico dado por el número de ejes patrón de 80 KN durante el periodo de proyecto.

Es de destacar que en el caso del uso de las ecuaciones anteriores (6 y 7) para verificar la condición de un pavimento se reemplaza Es por EsgCalc (según la ecuación 8) teniendo en cuenta que se trata de un pavimento en servicio.

Para las mediciones de campo del cuenco de deflexión realizadas con la viga Benkelman se determinan, a partir de la deflexión máxima y el radio de curvatura, la estructura del pavimento equivalente y el módulo de la subrasante utilizando las siguientes ecuaciones.

$$
\begin{gathered}
\text { EsgCal }=\left(51386.227 * D_{c}{ }^{-0.674}\right) * R d c^{-\left(0.350 * D_{c}{ }^{0.368}\right)} \\
E p C a l=\left(3.144 * D_{c}^{0.028}\right) * R d c^{-\left(802.2 * D_{c}{ }^{1.050}\right)} \\
h C a l=\left(0.007 * D_{c}{ }^{0.324}\right) * R d c^{\left(0.169 * D_{c}^{0.368}\right)}
\end{gathered}
$$

Siendo:

Dc: Deflexión característica;

Rdc:- Radio de curvatura;

EsgCal: Módulo de la subrasante calculado;

EpCal: Módulo del pavimento equivalente calculado;

hCal: Espesor del pavimento equivalente calculado.

La deflexión característica es el parámetro que define las características del tramo homogéneo y se calcula según la ecuación referenciada siguiente: [16]

$$
D_{c}=\left(\sum_{i}^{n} \frac{D i}{n}+z * \sqrt{\sum_{i}^{n} \frac{\left(D i-\sum_{i}^{n} \frac{D i}{n}\right)^{2}}{n-1}}\right) * F_{t} * F_{h} * F_{c}
$$

Siendo:

Dc: Deflexión característica del tramo (100-1 mm);

Di: Deflexión en el punto i (100-1 mm);

n: Número de puntos medidos;

Ft: Factor de corrección por temperatura;

Fh: Factor de corrección por humedad;

Fh: Factor de corrección por carga;

z: Valor estándar para la probabilidad de cálculo.

Los valores derivados de las ecuaciones 6 y 7 , además los valores procedentes de las ecuaciones 8,9 y 10 se combinan para determinar la condición estructural de la vía (CEV), en este caso el pavimento necesita refuerzo si se cumple la siguiente condición:

$$
C E V=\frac{\mathrm{EpCal}^{*} \mathrm{hCal}}{\mathrm{E}^{*} \mathrm{~h}}<1
$$

El espesor de refuerzo necesario para la rehabilitación del pavimento se calcula utilizando la siguiente ecuación:

$$
\mathrm{H}=\frac{\mathrm{E}^{*} \mathrm{~h}-\mathrm{EpCal}{ }^{*} \mathrm{hCal}}{\mathrm{Ep}}
$$


Siendo:

H: Espesor de la capa de refuerzo.

Ep: Módulo de la capa de refuerzo que se utilizará (fue adoptado 1000 MPa para este trabajo según el módulo dinámico de la mezcla determinado para las condiciones de Angola según el método de la SHELL, [17].

Aquí E y h se calculan con las ecuaciones 6 y 7 reemplazando Es por EsgCalc teniendo en cuenta que se trata de un pavimento en servicio.

\section{Expresiones matemáticas para ser usadas a partir de datos de campo obtenidos con el Deflectómetro de Impacto}

Por otro lado las mediciones de campo de la deflexión con el Deflectómetro de Impacto permiten obtener una mayor y más precisa información obteniéndose parámetros del cuenco de deflexión tales como el Índice de la Capa Superficial (ICS), el Índide de la Capa Intermedia(ICI), el Índice de la Capa Baja (ICB), la deflexion máxima admisible (D0) y la deflexión medida a una distancia de $120 \mathrm{~mm}$ del punto de máxima deflexión (D120), lo cual permite estimar el módulo de la subrasante por medio de la siguiente expresión matemática obtenida a partir de la metodología propuesta.

$$
E s f=103.4^{*} D 120^{-1.0}
$$

Siendo:

D 120 es la deflexión medida a $120 \mathrm{~cm}$ del punto central de aplicación de la carga.

Para realizar el diagnóstico del pavimento se precisan las condiciones de la capa de superficie, la capa intermedia y la capa baja respectivamente, sobre la base del tráfico previsto, al utilizar las ecuaciones siguientes:

$$
\begin{aligned}
& I C S_{A d m}=20.947 * e^{-0.005 * E s f} * N^{-0.1} \\
& I C B_{\text {Adm }}=-0.257 * N^{-0.26 * E S f+9.541 * N^{0.18}} \\
& I C I A d m=-0.218 * N^{-0.29} * E s f+12.8 * N^{-0.18} \\
& D 0 A d m=(2.06 * \ln (E s f)-8.936) * \operatorname{Ln}(N)+121.2 * \operatorname{Esf}(-0.39) \\
& \left(h^{*} E p\right)_{A d m}=314.7 * N^{0.123} * E S f^{-(-0.003 * \mathrm{~N}+0.189)}
\end{aligned}
$$

Siendo:

$\mathrm{ICS}_{\mathrm{Adm}}:$ Índice de la capa superficial admisible;

$\mathrm{ICB}_{\mathrm{Adm}}$ : Índice de la capa baja admisible
$\mathrm{ICI}_{\mathrm{Adm}}:$ Índide de la capa intermedia admisible;

$\mathrm{Do}_{\mathrm{Adm}}$ : Deflexion máxima admisible

$\mathrm{h}{ }^{*} \mathrm{Ep}_{\mathrm{Adm}}$ : Producto espesor módulo de la capa equivalente admisible;

N- Tráfico previsto

Esf: Módulo de la subrasante obtenido con la ecuacion 14.

Teniendo en cuenta la posibilidad que brinda el deflectómetro de evaluar todas y cada una de las capas del pavimento entonces para la desición con respecto a la intervención se consideran los siguientes aspectos, es necesario realizar la reconstrucion total si el valor del módulo de la subrasante es menor que 2 o si el valor del ICB es mayor que el admisible (deficiencia en la capa baja).

Se considera además necesaria la reconstrucion parcial si el valor del ICI es mayor que el valor admisible (deficiencia en la capa intermedia) o si el valor del ICS es mayor que el admisible (deficiencia en la capa de superficie).

Se determina el producto del módulo del pavimento por el espesor de la capa equivalente utilizando la siguiente ecuación:

$$
h \text { Calc } * \text { EpCalc }=1201.074 * e^{0.034^{*} D_{0}} * e^{\left(-\left(-0.003^{*} D 0+0.011\right)^{*} E s s\right)}
$$

Siendo:

hCal * EpCal: Estructura equivalente calculada,

D0: Deflexión máxima.

El pavimento necesita intervención si al calcular el parámetro denominado condición estructural de la vía (CEV) se cumple la siguiente condición:

$$
C E V=\frac{E p C a l c^{*} h \text { Calc }}{\left(h^{*} E p\right)_{A d m}}<1
$$

El espesor de refuerzo se calcula utilizando la siguiente ecuación:

$$
\mathrm{H}=\frac{\left(h^{*} E p\right)_{A d m-\mathrm{EpCal} * \mathrm{hCaE}}}{\mathrm{EP}}
$$

Siendo:

$\mathrm{H}$ : Espesor de la capa de refuerzo;

Ep: Módulo de la capa de refuerzo.

(hCal"EpCalc): Estructura equivalente calculada

En general independientemente del instrumento de medición utilizado si el espesor del refuerzo del pavimento $(\mathrm{H})$ es menor 
que 3 se considera para la intervención que es solamente necesaria la conservación o mantenimiento, si varía entre 3 y 12 se considera necesario realizar el refuerzo de la estructura y para valores mayores que 12 se considera la reconstrucción según criterios adaptados de la literatura referenciada [18].

\section{Caso de Estudio}

En el caso que se analiza se presentan los resultados de la evaluación realizada en el tramo de la carretera EN-120 perteneciente a la red fundamental de carreteras de Angola, entre las localidades de Dondo y desvío da Munenga, con una longitud de $47.8 \mathrm{~km}$.

El desarrollo del trabajo incluye la ejecución de la medición de la deflexión con la viga Benkelman y con el Deflectómetro de Impacto, dada la división de la carretera en tramos homogéneos se trabaja con 68 tramos. Se aplican las ecuaciones propuestas y los resultados se comparan con la recomendación del catálogo SATCC.

Para la determinación del tráfico $(\mathrm{N})$ se realizó el conteo de vehículos por un periodo de 24 horas [18] estableciendo el valor del mismo en 1*106 ejes equivalentes de $80 \mathrm{KN}$.

- Evaluación a partir de las mediciones con la viga Benkelman

Para la evaluación con la viga Benkelman se utilizó un vehículo pesado, con carga de $8000 \mathrm{~kg}$ y eje trasero simple con ruedas dobles. Se calibraron los neumáticos con una presión de inflado de 0.56MPa. Los resultados de las mediciones y los correspondientes cálculos según las ecuaciones de la 6 a la 12 anteriormente descritas así como la recomendación en cuanto a la intervención en cada tramo sobre la base de la condición estructural de la vía y el valor de la capa de refuerzo $(\mathrm{H})$ se muestran en la tabla 1.
- Evaluación a partir de las mediciones con el Deflectómetro de Impacto

Para la realización de las mediciones con el Deflectómetro de Impacto se utilizó una carga normalizada de $40 \mathrm{KN}$ aplicada sobre una placa de $30 \mathrm{~cm}$ de diámetro [19], en la tabla 2 se presentan los resultados de las mediciones así como los cálculos realizados con las ecuaciones previstas (ecuaciones 14 a la 19).

- Evaluación a partir de las recomendaciones de la norma SATCC

De acuerdo con la norma SATCC (2001) los pavimentos se clasifican en diversas categorías, en este caso concreto se ubica como un pavimento que pertenece a la categoría $C$, para esta categoría la condición del pavimento se define considerándose adecuado cuando la deflexión es menor o igual a 65 y es crítica cuando la deflexión es mayor o igual a 135.

En el punto 3.4.2 de la referida norma relacionado con los métodos de diseño de rehabilitación se recomiendan el empleo de diversos métodos de cálculo siendo el proyectista el responsable de la selección del que considere adecuado para su aplicación concreta. Se consideran los siguientes métodos, el del penetrómetro dinámico de cono (DCP por sus siglas en ingles) y el método propuesto por el Instituto del Asfalto y el mecánico empírico de Sudáfrica, además el método del Laboratorio de Investigaciones en Transporte (Reino Unido) reconocido por sus siglas en ingles TRL y el de la Shell. Para el caso de la carretera en estudio se verifica y clasifica para cada tramo homogéneo en correspondencia con el valor calculado de la deflexión según la metodología de la TRL, por considerarse este último método más adecuado para países tropicales con una aplicación muy objetiva.

La norma TRL presenta una ecuación que permite calcular el espesor de refuerzo basado en la deflexión del pavimento para refuerzo con mezcla asfáltica densa, para deflexiones medidas con una carga de $62.3 \mathrm{kN}$ :

Tabla 1. Cálculos realizados a partir de las mediciones con la viga Benkelman

\begin{tabular}{|c|c|c|c|c|c|c|c|c|c|c|}
\hline \multirow{2}{*}{ Tramo } & $\mathbf{D C}$ & $\mathbf{R d c}$ & $\mathbf{E s g C a l}$ & $\mathbf{E p C a l}$ & $\mathbf{h C a l}$ & $\mathbf{h}$ & $\mathbf{E}$ & CEV & H & $\begin{array}{c}\text { Propuesta de } \\
\text { intervención }\end{array}$ \\
\cline { 2 - 13 } & $\mathbf{X 1 0 0 \mathbf { m m }}$ & $\mathbf{m}$ & $\mathbf{M P a} / \mathbf{1 0}$ & $\mathbf{M P a}$ & $\mathbf{M}$ & $\mathbf{m}$ & $\mathbf{M P a}$ & - & $\mathbf{m m}$ & $\mathbf{C}$ \\
\hline 1 & 54.6 & 106 & 3 & 361 & 0.8 & 0.63 & 485 & 0.7 & 0.02 & 0.07 \\
\hline 2 & 77.8 & 77 & 2 & 264 & 1.09 & 0.86 & 415 & 0.6 & REF \\
\hline 13 & 70.7 & 87 & 2 & 300 & 1.04 & 0.82 & 426 & 0.7 & 0.04 & REF \\
\hline 25 & 71.2 & 78 & 2 & 269 & 0.96 & 0.76 & 442 & 0.6 & 0.08 & REF \\
\hline 49 & 101.4 & 56 & 1 & 193 & 1.29 & 1.01 & 384 & 0.5 & 0.14 & REC \\
\hline 50 & 115.1 & 42 & 1 & 145 & 1.22 & 0.96 & 394 & 0.4 & 0.20 & REC \\
\hline 61 & 62.1 & 100 & 2 & 343 & 0.94 & 0.74 & 448 & 0.8 & 0.01 & C \\
\hline 68 & 67.6 & 83 & 2 & 285 & 0.93 & 0.73 & 450 & 0.6 & 0.06 & REF \\
\hline
\end{tabular}

C - Conservación, REF - Refuerzo, REC - Reconstrucción 
Tabla 2. Cálculos realizados a partir de las mediciones con el Deflectómetro de Impacto

\begin{tabular}{|c|c|c|c|c|c|c|c|c|c|c|c|c|c|c|c|}
\hline \multirow{2}{*}{$\begin{array}{l}\text { 을 } \\
\text { 롤 }\end{array}$} & \multirow[b]{2}{*}{0} & \multicolumn{3}{|c|}{ Deflexiones medidas } & \multirow[b]{2}{*}{120} & & \multicolumn{3}{|c|}{ Medidos y calculados } & \multirow[b]{2}{*}{$\begin{array}{c}\text { Estruct. } \\
\text { equiv } \\
\text { Calc. }\end{array}$} & \multirow[b]{2}{*}{ CEV } & \multirow[b]{2}{*}{ H } & \multicolumn{2}{|c|}{$\begin{array}{l}\text { Propuesta Intervención } \\
\text { según: }\end{array}$} \\
\hline & & 30 & 60 & 90 & & Esf & ICS & ICI & ICB & Do & & & & H & $\begin{array}{c}\text { Parámetros } \\
\text { del cuenco } \\
\text { Deflexión }\end{array}$ \\
\hline 1 & 49 & 18 & 8 & 4 & 1 & 8 & -11 & 1 & 3 & 5 & 178 & 1.2 & 0 & C & REF \\
\hline 2 & 81 & 28 & 11 & 6 & 3 & 4 & -32 & -5 & 3 & -7 & 753 & 0.3 & 0.05 & REF & RECP \\
\hline 25 & 72 & 23 & 8 & 4 & 2 & 6 & -28 & -4 & 4 & -13 & 339 & 0.7 & 0.01 & C & RECP \\
\hline 49 & 104 & 46 & 19 & 10 & 4 & 2 & -37 & -14 & 0 & -16 & 1922 & 0.1 & 0.17 & REC & RECT \\
\hline 50 & 120 & 51 & 20 & 11 & 5 & 2 & -48 & -18 & 0 & -27 & 3397 & 0.1 & 0.31 & REC & RECT \\
\hline 61 & 84 & 33 & 12 & 5 & 2 & 5 & -30 & -9 & 2 & -18 & 597 & 0.4 & 0.04 & REF & RECP \\
\hline 68 & 68 & 27 & 12 & 8 & 4 & 2 & -20 & -3 & 4 & 19 & 731 & 0.4 & 0.05 & REF & RECP \\
\hline
\end{tabular}

C - Conservación, REF - Refuerzo, REC - Reconstrucción. RECP (Parcial) RECT (Total)

$$
T=\frac{0.036+0.818^{*} D_{T}^{ \pm}-D_{d}}{0.0027 * D_{d}}
$$

Siendo:

Dt: Deflexión de diseño;

Dd: Deflexión característica;

T: Espesor de refuerzo.

Como se muestra en la tabla 3 en este caso no se verifica ningún tramo con valores de deflexión superiores a 125 lo que indica que en términos de evaluación con la propuesta en la norma SATCC el pavimento presenta buenas condiciones para todos los tramos en términos deflectométricos. En términos de refuerzo se presentan los valores calculados $(\mathrm{T})$ según los criterios de la TRL y se aplica para evaluar en términos de intervención el mismo criterio aplicado para todo el trabajo de modo que es posible realizar la comparación en base al espesor de refuerzo con los espesores obtenidos por las ecuaciones desarrolladas en el presente trabajo.

En general la evaluación de los resultados permite inferir que las ecuaciones propuestas son de adecuados resultados tanto si se tienen datos procedentes de mediciones realizadas con la viga Benkelman como con el Deflectómetro de Impacto, con una coincidencia en cuanto a la decisión a tomar para la intervención de más del $85 \%$ de los tramos evaluados por cada uno de los dos métodos.

Es de destacar que en el caso de la aplicación del deflectómetro permite la evaluación a través del ICI, del ICS y del ICB de todas las capas de la estructura a partir de los cuencos de deflexión medidos y la aplicación de las ecuaciones desarrolladas. No obstante el uso de la viga Benkelman puede resultar adecuado al emplear las ecuaciones propuestas cuando no está disponible un instrumento más preciso.

Utilizando los índices ICS, ICI y ICB se verifica que todos los tramos necesitan de refuerzo los que es lógico pues en el pavimento evaluado la capa asfáltica se construyó con un tratamiento superficial con espesores entre 1,50 y $2,5 \mathrm{~cm}$, mientras que, en la norma SATCC para un tráfico de $1 * 106$ ejes equivalentes de $8000 \mathrm{~kg}$ el espesor debería ser de $5 \mathrm{~cm}$ de hormigón asfáltico.

Los tramos que necesitan refuerzo están en relación con un valor bajo de ICI, lo que indica que la base no es suficiente para soportar el tráfico previsto debido a la capa asfáltica utilizada. Se confirma para los tramos con valores bajos del índice ICB que es necesario su reconstrucción.

Tabla 3. Cálculos realizados a partir de la norma SATCC usando la ecuación recomendada de Ia TRL

\begin{tabular}{|c|c|c|c|c|c|c|}
\hline \multirow{2}{*}{ Tramo } & $\mathbf{D c 8 0}$ & $\mathbf{D c 6 5}$ & $\mathbf{D T}$ & $\mathbf{D d}$ & $\mathbf{T}$ & \multirow{2}{*}{$\begin{array}{c}\text { Propuesta de } \\
\text { Intervención }\end{array}$} \\
\cline { 2 - 6 } & $\mathbf{x 1 0 0 \mathbf { m m }}$ & $\mathbf{x 1 0 0 \mathbf { m m }}$ & $\mathbf{M m}$ & $\mathbf{m m}$ & $\mathbf{m m}$ & $\mathbf{C}$ \\
\hline 1 & 54.64 & 42.36 & 0.42 & 0.40 & -15 & REF \\
\hline 2 & 77.8 & 60.37 & 0.60 & 0.40 & 80 & REF \\
\hline 13 & 70.7 & 54.85 & 0.55 & 0.40 & 57 & REF \\
\hline 25 & 71.2 & 55.24 & 0.55 & 0.40 & 59 & REC \\
\hline 49 & 101.4 & 78.73 & 0.79 & 0.40 & 132 & REC \\
\hline 50 & 115.1 & 89.39 & 0.89 & 0.40 & 152 & C \\
\hline 61 & 62.1 & 48.16 & 0.48 & 0.40 & 23 & REF \\
\hline 68 & 67.6 & 52.43 & 0.52 & 0.40 & 46 & \\
\hline
\end{tabular}

C - Conservación, REF - Refuerzo, REC - Reconstrucción 
Sin embargo el empleo de las recomendaciones de la SATCC presenta inconvenientes pues el uso de los catálogos y gráficas no permite una mejor caracterización del estado del pavimento, además sólo es posible obtener el espesor de refuerzo a partir de la deflexión máxima, todo lo cual se mejora con el uso de las ecuaciones planteadas que permiten evaluar la estructura del pavimento y la subrasante, además resultan más precisas al no considerar intervalos sino que es posible la introducción de los datos reales y la evaluación para cada tramo en función del tráfico y del módulo de la subrasante.

\section{CONCLUSIONES}

La metodología propuesta permite de forma precisa evaluar y determinar la condición del pavimento en su conjunto o en cada una de sus capas, reduciendo significativamente la necesidad de realización de sondeos lo que incrementa la rapidez en la obtención de los resultados, a su vez se optimiza la adopción de la mejor solución en cuanto a la intervención necesaria para el mejoramiento del pavimento en uso.

Las ecuaciones propuestas pueden ser introducidas en un sencillo programa de computación de modo que con un reducido número de parámetros de entrada se puede obtener de forma rápida y precisa el diseño de la estructura completa del pavimento tanto para nuevas instalaciones como para la rehabilitación o reconstrucción de las existentes.

Las expresiones matemáticas obtenidas para la determinación de los índices de ISC, ICI e ICB demuestran que los mismos dependen del módulo de la subrasante y del tráfico. La aplicación de las expresiones propuestas manifiesta, en relación con la norma SATCC en uso, una importante economía de recursos materiales.

\section{REFERENCIAS BIBLIOGRAFICAS}

1. Nóbrega, E.S., Comparação entre métodos de retroanálise em pavimentos asfálticos., in COPPE 2003, Universidade Federal do Rio de Janeiro, : Rio de Janeiro.

2. SADC (1996) Protocol on Transport, Communications and Meteorology.

3. Borges, C.B.S., Estudo comparativo entre medidas de deflexão com viga benkelman e fwd em pavimentos da malha rodoviária estadual de Santa Catarina, in COPPE. 2001, Universidade Federal de Santa Catarina: Florianópolis.

4. Torres, M.S., J.I.M. Rodríguez, and L.G.P. Guevara, Posibles factores de incidencia en el valor de las mediciones de deflexión de un pavimento. 2002.

5. Saltan, M., M. Karaşahin, and S. Terzi On the Backcalculation of Flexible Road Pavement Material Properties and New Methods

6. Albernaz, C.A.V., Método Simplificado de Retroanálise de Módulos de Resiliência de Pavimentos flexiveis a partir da Bacia de Deflexão, in COPPE. 1997, Universidade federal do Rio de Janeiro: Rio de Janeiro.

7. Neto, C.A. Aplicabilidade da norma satcc na elaboração de projectos de reabilitação de pavimentos flexíveis de estradas em angola. in XV CILA - Congresso IberoLatinoamericano do Asfalto, 2009. Lisboa.

8. Macêdo, J.A.G.d., interpretação de ensaios deflectométricos para avaliação de estructuras de pavimentos flexiveis, in COPPE. 1996, univbersidade federal do Rio de Janeiro: Rio de Janeiro.

9. Río, R.C.d. and J.F.R. Romero, Evaluación estructural de firmes de carreteras. El área del cuenco de deflexiones . El módulo de la explanada, Á.d.A.d.F.A.i. consultores, Editor. 2001

10. Broutin, M., Assessment of flexible airfield pavement using heavy weight deflectómeters (dvelopment of a FEM dynamical time-domain analisys for backcalculation of strctural propertis, in Ecole national des ponts et chaussées. 2010: Paris.

11. Horak, E., et al., Correlation Study With the Light Weight Deflectometer in South Africa, in.27a Conferencia de Transporte de Sudáfrica SATC, Editor. 2008. p. 304-312.

12. SATCC, Code of Practice for the Design of Road Pavements, D.o.R.a.T. Technology, Editor. 2001, CSIR: Pretoria.

13. Franco, F.A.C.d.P., Método de dimensionamiento mecanístico empírico de pavimentos asfálticos - SisPav. [2007, Universidade Federal do Rio de Janeiro, COPPE.: Rio de Janeiro.

14. Vila, J.A.T., Diseño de pavimentos para carreteras y aeropuertos, ed. I. Facultad de Hidraulica y viales. Vol. I y II. 1985, La Habana: Departamento de carreteras y aeropuertos.

15. AASHTO, A.A.o.S.H.a.T.O.e.c.-. Mechanistic-Empirical Pavement Design Guide - A Manual of Practice, A.A.o.S.H.a.T. Officials, Editor. 2008.

16. Norma_6.3-IC, Rehabilitación de firmes, de la Instrucción de carreteras., D.g.d.C.m.d.f.d. España, Editor. 2003: Madrid.

17. Silva, J.d.A.A.e., et al. Estudo sobre o comportamento fisico e mecânico de misturas asfálticas com faixas granulométricas preconizadas para revestimentos de aeródromos. in XVI CILA - Congreso Ibero-Latinoamericano del Asfalto. 2011. Rio de Janeiro Instituto Brasileiro de Petróleo, Gás e Biocombustíveis - IBP

18. DNIT, Manual de Restauração de pavimentos asfálticos D.N.d.I.-E.d.T.D.d.P.e. Pesquisa, Editor. 2006, Instituto de Pesquisas Rodoviárias: Rio de Janeiro.

19. Rodrigues, R.M., Engenharia de Pavimentos_Parte I - Projeto de Pavimentos. 2007. I and II(Instituto tecnológico de aeronáutica: divisão de engenharia de infra-estrutura aeronáutica). 